\title{
On Improving Ratio/Product Estimator by Ratio/Product-cum-Mean-per-Unit Estimator Targeting More Efficient Use of Auxiliary Information
}

\author{
Angela Shirley, Ashok Sahai, and Isaac Dialsingh \\ Department of Mathematics and Statistics, Faculty of Science and Technology, The University of the West Indies St Augustine, \\ St. Augustine, Trinidad and Tobago
}

Correspondence should be addressed to Angela Shirley; angela.shirley@sta.uwi.edu

Received 2 August 2014; Accepted 28 August 2014; Published 23 September 2014

Academic Editor: Aera Thavaneswaran

Copyright (C) 2014 Angela Shirley et al. This is an open access article distributed under the Creative Commons Attribution License, which permits unrestricted use, distribution, and reproduction in any medium, provided the original work is properly cited.

\begin{abstract}
To achieve a more efficient use of auxiliary information we propose single-parameter ratio/product-cum-mean-per-unit estimators for a finite population mean in a simple random sample without replacement when the magnitude of the correlation coefficient is not very high (less than or equal to 0.7 ). The first order large sample approximation to the bias and the mean square error of our proposed estimators are obtained. We use simulation to compare our estimators with the well-known sample mean, ratio, and product estimators, as well as the classical linear regression estimator for efficient use of auxiliary information. The results are conforming to our motivating aim behind our proposition.
\end{abstract}

\section{Introduction and Notation}

This paper addresses the problem of efficiently estimating the population mean, using auxiliary information. A fairly large simple random sample of size $n$ is selected without replacement from, say, a large bivariate population of size $N$ (which could, reasonably, be thought to have come from a normal superpopulation), with the sampling fraction $f=$ $n / N, N \gg n$, so that $f$ is negligible. Quite often, we have surveys where some auxiliary variable $X$ may be relatively less expensive to observe than the main variable $Y$. In order to have a survey estimate of the population mean $\bar{Y}$ of the main variable, assuming knowledge of the population mean $\bar{X}$ of the auxiliary variable, the following estimators are well known.

The ratio estimator:

$$
\widehat{\bar{Y}}_{R}=\widehat{R} \bar{X} .
$$

The product estimator:

$$
\hat{\bar{Y}}_{P}=\frac{\widehat{P}}{\bar{X}} .
$$

Here $\widehat{R}=\bar{y} / \bar{x}$ is the estimate of the ratio of the population means and $\widehat{P}=\bar{y} \cdot \bar{x}$ is the estimate of the product of the population means, $\bar{y}$ and $\bar{x}$ being unweighted sample means of the two variables, respectively. Usually, the variability of $\bar{x}$ is less than that of $\bar{y}$.

It is straightforward to derive first order approximations to the bias and mean square error of these estimators. Let $C_{Y}=S_{Y} / \bar{Y}$ and $C_{X}=S_{X} / \bar{X}$ be the population coefficients of variation of $Y$ and $X$, respectively, where

$$
\begin{aligned}
& S_{Y}^{2}=(N-1)^{-1} \sum\left(Y_{i}-\bar{Y}\right)^{2}, \\
& S_{X}^{2}=(N-1)^{-1} \sum\left(X_{i}-\bar{X}\right)^{2}
\end{aligned}
$$

are the population variances of $Y$ and $X$, respectively. Let $\bar{y}=\bar{Y}(1+e)$ and $\bar{x}=\bar{X}\left(1+e^{\prime}\right)$, where the errors $e$ and $e^{\prime}$ can be positive or negative, so that $E(e)=E\left(e^{\prime}\right)=0$. It is known that, for simple random sample without replacement, $\operatorname{Var}(e)=(1-f) C_{Y}^{2} / n, \operatorname{Var}\left(e^{\prime}\right)=(1-f) C_{X}^{2} / n$, and $\operatorname{Cov}\left(e, e^{\prime}\right)=$ $(1-f) \rho C_{X} C_{Y} / n$ where $\rho$ is the correlation coefficient between the variables (P. V. Sukhatme and B. V. Sukhatme [1]). Further, to validate our first order large sample approximations, we 
assume that the sample is large enough to make $|e|$ and $\left|e^{\prime}\right|$ so small that the terms involving $e$ and/or $e^{\prime}$ to a degree higher than two are negligible, an assumption which is not unrealistic.

Substituting the expressions for $\bar{y}$ and $\bar{x}$ in terms of $e$ and $e^{\prime}$ in (1) we have

$$
\widehat{\bar{Y}}_{R}=(1+e)\left(1+e^{\prime}\right)^{-1} \bar{Y}
$$

Assuming that $\left|e^{\prime}\right|<1$, we expand $\left(1+e^{\prime}\right)^{-1}$ to obtain

$$
\operatorname{Bias}\left(\widehat{\bar{Y}}_{R}\right)=\frac{(1-f)}{n} \bar{Y} C_{X}\left(C_{X}-\rho C_{Y}\right),
$$

up to the first order of approximation, $\mathrm{O}\left(\frac{1}{n}\right)$.

Since $(1-f) / n \rightarrow 0$ as $n \rightarrow \infty$, we have that the ratio estimator is asymptotically unbiased up to $\mathrm{O}(1 / n)$. Similarly we have that the product estimator is asymptotically unbiased (Murthy [2]). Also,

$$
\operatorname{MSE}\left(\hat{\bar{Y}}_{R}\right)=E\left(\hat{\bar{Y}}_{R}-\bar{Y}\right)^{2} \approx E\left[\hat{\bar{Y}}^{2}\left(e^{2}+e^{\prime 2}-2 e e^{\prime}\right)\right],
$$

up to the first order of approximation

$$
\begin{aligned}
& =\left\{\bar{Y}^{2} \frac{(1-f)}{n}\right\}\left\{C_{Y}^{2}+C_{X}^{2}-2 \rho C_{X} C_{Y}\right\} \\
& =\operatorname{Var}(\bar{y})+\frac{(1-f)}{n} \bar{Y}^{2}\left\{C_{X}^{2}-2 \rho C_{X} C_{Y}\right\} .
\end{aligned}
$$

Thus up to order $\mathrm{O}(1 / n)$ of approximation, $\operatorname{MSE}\left(\hat{\bar{Y}}_{R}\right)<$ $\operatorname{Var}(\bar{y})$ if and only if $C_{X}^{2}-2 \rho C_{X} C_{Y}<0$, or if and only if $C>1 / 2$, where $C=\rho C_{Y} / C_{X}$. (It is worth noting here that because of long association with the experimental data, $C$ is guessable.) Similarly we have $\operatorname{MSE}\left(\hat{\bar{Y}}_{P}\right)<\operatorname{Var}(\bar{y})$ if and only if $C<-1 / 2$ (Murthy [2]). Thus the ratio and product estimators are relatively more efficient than the usual unbiased estimator (u.u.e) sample mean when $C>1 / 2$ and $C<-1 / 2$, respectively. Consequently, $\widehat{\bar{Y}}_{R} / \widehat{\bar{Y}}_{P}$ fail to improve $\bar{y}$ (by using auxiliary information) when $-1 / 2 \leq C \leq 1 / 2$.

Also we cannot ignore the classically well-known linear regression estimator, say $\widehat{\bar{Y}}_{\mathrm{LR}}$ :

$$
\begin{gathered}
\hat{\bar{Y}}_{\mathrm{LR}}=\bar{y}+\widehat{\beta}(\bar{X}-\bar{x}), \\
\text { where } \widehat{\beta}=\frac{\sum_{i=1}^{n}\left(y_{i}-\bar{y}\right)\left(x_{i}-\bar{x}\right)}{\sum_{i=1}^{n}\left(x_{i}-\bar{x}\right)^{2}} .
\end{gathered}
$$

If we recall the ANOVA of linear regression analysis, we must remember that the residual sum of squares for $\widehat{\bar{Y}}_{\mathrm{LR}}$ is $S_{Y}^{2}(1-$ $\rho^{2}$ ) (Cochran [3]). Thus when $|\rho|$ is high (say $\rho>0.7$ or $\rho<$ - 0.7), linear regression estimator is most likely to be more efficient than ratio/product estimators in using the auxiliary information (via auxiliary variable $X$ ). We aim at improving use of auxiliary information on $\bar{y}$ when $-1 / 2 \leq C \leq 1 / 2 ; \widehat{\bar{Y}}_{R}$ when $C>1 / 2 ; \widehat{\bar{Y}}_{P}$ when $C<-1 / 2$; and $\widehat{\bar{Y}}_{\mathrm{LR}}$ when $|\rho| \leq 0.7$.

\section{Our Proposed Estimators}

Because $\hat{\bar{Y}}_{R}$ and $\hat{\bar{Y}}_{P}$ are relatively more efficient than $\bar{y}$ when $C>1 / 2$ and $C<-1 / 2$, respectively, we try the following single parameter linear combinations of $\widehat{\bar{Y}}_{R}$ and $-\bar{y}$, as well as $\widehat{\bar{Y}}_{P}$ and $-\bar{y}$ to propose the estimators:

(i) Shirley-Sahai-Dialsingh-ratio-cum-mean, say $\widehat{\bar{Y}}_{\text {SSDR }}$ :

$$
\widehat{\bar{Y}}_{\mathrm{SSDR}}=(1+\theta) \hat{\bar{Y}}_{R}-\theta \bar{y} \text {. }
$$

(ii) Shirley-Sahai-Dialsingh-product-cum-mean, say $\widehat{\bar{Y}}_{\text {SSDP }}$ :

$$
\widehat{\bar{Y}}_{\text {SSDP }}=(1+\theta) \hat{\bar{Y}}_{P}-\theta \bar{y} \text {. }
$$

In (8) and (9), $\theta$ is the design parameter for our proposed estimators to be assigned an optimal value, for example, so as to minimize the first order of MSE, $M_{1}(\circ)$, as in our case. Note that when $\theta=0, \widehat{\bar{Y}}_{\mathrm{SSDR}}=\widehat{\bar{Y}}_{R}$ and $\widehat{\bar{Y}}_{\mathrm{SSDP}}=\widehat{\bar{Y}}_{P}$. As remarked earlier, quite often a good guess of $C$ is available from which we can give a suitable value to $\theta$.

\section{Sampling Bias and Mean Square Error of the Proposed Estimators}

We derive the first order approximation, $B_{1}\left(\hat{\bar{Y}}_{\mathrm{SSDR}}\right)$, to the bias of $\widehat{\bar{Y}}_{\text {SSDR }}$. Using the notation introduced in Section 1 and substituting the expressions for $\bar{y}$ and $\bar{x}$ in terms of $e$ and $e^{\prime}$ in (8) we have

$$
\widehat{\bar{Y}}_{\mathrm{SSDR}}=\bar{Y}(1+e)\left(1-\theta e^{\prime}\right)\left(1+e^{\prime}\right)^{-1} .
$$

It is realistic practically to suppose that $\left|e^{\prime}\right|<1$ so that $(1+$ $\left.e^{\prime}\right)^{-1}$ is expandable. Then to the first order of approximation $\{\mathrm{O}(1 / n)\}$, the bias of $\widehat{\bar{Y}}_{\text {SSDR }}$ is given by

$$
\begin{aligned}
B_{1}\left(\hat{\bar{Y}}_{\mathrm{SSDR}}\right) & =E\left(\hat{\bar{Y}}_{\mathrm{SSDR}}-\bar{Y}\right) \\
& \approx \bar{Y}\left\{(1-\theta) E\left(e^{\prime 2}\right)-(1+\theta) E\left(e e^{\prime}\right)\right\} \\
& =B_{0}\{a(2-F)-\rho F\},
\end{aligned}
$$

where $a=C_{X} / C_{Y}, F=1+\theta$, and $B_{0}=a(1-f) \bar{Y} C_{Y}^{2} / n . B_{0} \rightarrow$ 0 , as $n \rightarrow \infty$; therefore, $\hat{\bar{Y}}_{\mathrm{SSDR}}$ is asymptotically unbiased up to $\mathrm{O}(1 / n)$.

To compute the MSE of $\hat{\bar{Y}}_{\text {SSDR }}$ we have

$$
M_{1}\left(\hat{\bar{Y}}_{\mathrm{SSDR}}\right)=E\left(\hat{\bar{Y}}_{\mathrm{SSDR}}-\bar{Y}\right)^{2} \approx \bar{Y}^{2} E\left\{\left(e-F e^{\prime}\right)^{2}\right\},
$$

up to the first order of approximation $\left\{\mathrm{O}\left(\frac{1}{n}\right)\right\}$

$$
\begin{aligned}
& =\left\{\bar{Y}^{2} \frac{(1-f)}{n}\right\}\left\{C_{Y}^{2}+F^{2} C_{X}^{2}-2 F \rho C_{X} C_{Y}\right\} \\
& =V_{0}\left\{1+a^{2} F^{2}-2 a F \rho\right\},
\end{aligned}
$$

where $V_{0}=\operatorname{Var}(\bar{y})=((1-f) / n) S_{Y}^{2}$ and $S_{Y}=\bar{Y} C_{Y}$. 
TABLE 1: Relative efficiencies (in \%) of the estimators when $n=30$ and $R=0$.

\begin{tabular}{lcccc}
\hline$\rho$ & $C$ & $\hat{\bar{Y}}_{\mathrm{LR}}$ & $\hat{\bar{Y}}_{R}$ & $\widehat{\bar{Y}}_{\text {SSDR }}$ \\
\hline 0.1 & 0.225 & 97.00 & 89.71 & 100.88 \\
0.2 & 0.45 & 100.35 & 98.09 & 104.22 \\
0.3 & 0.675 & 105.26 & 106.32 & 109.10 \\
0.4 & 0.90 & 114.29 & 117.98 & 118.34 \\
0.5 & 1.125 & 128.73 & 133.07 & 133.58 \\
0.6 & 1.35 & 152.63 & 152.08 & 159.40 \\
0.7 & 1.575 & 186.79 & 172.17 & 193.33 \\
\hline
\end{tabular}

For large sample size, $M_{1}\left(\hat{\bar{Y}}_{\mathrm{SSDR}}\right)$ is minimum for $a F=\rho$. The optimal value of $F$ is thus $\rho / a=C$. If a good guess of $C$, say $C^{*}$, is available, we use $\theta=C^{*}-1$ in our proposed estimator (8), so that

$$
\widehat{\bar{Y}}_{\mathrm{SSDR}} \approx C^{*} \frac{\bar{y} \bar{X}}{\bar{x}}-\left(C^{*}-1\right) \bar{y} .
$$

We deduce the large sample approximation for bias of $\widehat{\bar{Y}}_{\text {SSDP }}$ in a similar manner:

$$
\begin{aligned}
\hat{\bar{Y}}_{\mathrm{SSDP}} & =\bar{Y}(1+e)\left(1+(1+\theta) e^{\prime}\right), \\
B_{1}\left(\widehat{\bar{Y}}_{\mathrm{SSDP}}\right) & =E\left(\widehat{\bar{Y}}_{\mathrm{SSDP}}-\bar{Y}\right)=\bar{Y}(1+\theta) E\left(e e^{\prime}\right) \\
& =B_{0} \rho F
\end{aligned}
$$

where $F$ and $B_{0}$ are as before. $B_{0} \rightarrow 0$, as $n \rightarrow \infty$; therefore $\widehat{\bar{Y}}_{\text {SSDP }}$ is asymptotically unbiased.

To compute the MSE of $\widehat{\bar{Y}}_{S S D P}$ we have

$$
M_{1}\left(\hat{\bar{Y}}_{S S D P}\right)=E\left(\hat{\bar{Y}}_{S S D P}-\bar{Y}\right)^{2} \approx \bar{Y}^{2} E\left\{\left(e+F e^{\prime}\right)^{2}\right\},
$$

up to the first order of approximation $\left\{\mathrm{O}\left(\frac{1}{n}\right)\right\}$

$$
\begin{aligned}
& =\left\{\bar{Y}^{2} \frac{(1-f)}{n}\right\}\left\{C_{Y}^{2}+F^{2} C_{X}^{2}+2 F \rho C_{X} C_{Y}\right\} \\
& =V_{0}\left\{1+a^{2} F^{2}+2 a F \rho\right\}, \\
& \text { where } V_{0}=\operatorname{Var}(\bar{y}) .
\end{aligned}
$$

Up to $\mathrm{O}(1 / n), M_{1}\left(\hat{\bar{Y}}_{\mathrm{SSDP}}\right)$ is a minimum for $a F=-\rho$. The optimal value of $F$ is thus $-\rho / a=-C$.

We use $\theta=-C^{*}-1$ in our proposed estimator (9), where $C^{*}$ is the guess of $C$. Thus,

$$
\widehat{\bar{Y}}_{\mathrm{SSDP}} \approx-C^{*} \frac{\overline{x y}}{\bar{X}}+\left(C^{*}+1\right) \bar{y} .
$$

TABLE 2: Relative efficiencies (in \%) of the estimators when $n=30$ and $R=0$.

\begin{tabular}{lcccc}
\hline$\rho$ & $C$ & $\hat{\bar{Y}}_{\mathrm{LR}}$ & $\hat{\bar{Y}}_{P}$ & $\hat{\bar{Y}}_{\text {SSDP }}$ \\
\hline-0.1 & -0.225 & 97.66 & 89.14 & 100.73 \\
-0.2 & $-0 . .45$ & 100.42 & 98.05 & 104.14 \\
-0.3 & -0.675 & 106.48 & 108.23 & 110.39 \\
-0.4 & -0.90 & 114.87 & 119.11 & 119.43 \\
-0.5 & -1.125 & 127.32 & 131.54 & 132.05 \\
-0.6 & -1.35 & 150.37 & 150.36 & 155.88 \\
-0.7 & -1.575 & 186.53 & 172.80 & 194.68 \\
\hline
\end{tabular}

\section{Comparison of the Estimators}

Apparently, no algebraic comparison of mean square errors is feasible. We, therefore, have a numerical setup under simulation to do so. Knowing $C$ exactly is seldom tenable in practice. Consequently, we have to assume the availability of a guess value of $C$, which we have called $C^{*}$, defined by $C^{*}=$ $C(1+R)$, where $R$ designates the quantum of relative under guess/overguess. We have taken the following $R$ values: 0 , $\pm 0.02, \pm 0.04, \pm 0.06, \pm 0.08$, and \pm 0.10 . We have also assumed that the parent population is very large, envisaged to have come from a superpopulation which is bivariate normal with the following parameters, therefore having the same parametric values:

$$
\begin{aligned}
& \bar{Y}=20.00, \\
& \bar{X}=15.00, \\
& S_{Y}=3.00, \\
& S_{X}=1.00 .
\end{aligned}
$$

Consequently we have used the software $\mathbf{R}$ [4] to calculate the MSEs of each of the following estimators:

$$
\begin{gathered}
\hat{\bar{Y}}_{R}=\widehat{R} \bar{X} ; \\
\widehat{\bar{Y}}_{P}=\frac{\widehat{P}}{\bar{X}} ; \\
\widehat{\bar{Y}}_{\mathrm{SSDR}}=(1+\theta) \hat{\bar{Y}}_{R}-\theta \bar{y} ; \\
\widehat{\bar{Y}}_{\mathrm{SSDP}}=(1+\theta) \hat{\bar{Y}}_{P}-\theta \bar{y}, \\
\widehat{\bar{Y}}_{\mathrm{LR}}=\bar{y}+\widehat{\beta}(\bar{X}-\bar{x}) .
\end{gathered}
$$

We use 10,000 replications of simulated sample sizes $n=30$, $40,60,80$, and 100 . Hence we have compared the efficiencies of these estimators relative to $\bar{y}$ by using

$$
\operatorname{REff}(\circ)=\frac{\operatorname{MSE}(\bar{y})}{\operatorname{MSE}(\circ)} \times 100 \% .
$$

Motivated by our desire to beat ratio/product estimators (implicitly, therefore $\widehat{\bar{Y}}_{\mathrm{LR}}$ also), we have, therefore, taken up 
TABLE 3: Relative efficiencies (in \%) of $\widehat{\bar{Y}}_{\mathrm{LR}}, \hat{\bar{Y}}_{R}$, and $\widehat{\bar{Y}}_{\mathrm{SSDR}}$ when $n=30$.

\begin{tabular}{|c|c|c|c|c|c|c|c|}
\hline$n=30$ & $\rho=0.1$ & $\rho=0.2$ & $\rho=0.3$ & $\rho=0.4$ & $\rho=0.5$ & $\rho=0.6$ & $\rho=0.7$ \\
\hline$\overline{\bar{Y}}_{\mathrm{LR}}$ & 97.00734 & 100.3539 & 105.2556 & 114.2872 & 128.7345 & 152.627 & 186.7908 \\
\hline$\overline{\bar{Y}}_{R}$ & 89.71481 & 98.09601 & 106.3240 & 117.9799 & 133.0705 & 152.0778 & 172.174 \\
\hline$R$ value & $\widehat{\bar{Y}}_{\mathrm{SSDR}}$ & $\widehat{\bar{Y}}_{\mathrm{SSDR}}$ & $\widehat{\bar{Y}}_{\mathrm{SSDR}}$ & $\widehat{\bar{Y}}_{\mathrm{SSDR}}$ & $\widehat{\bar{Y}}_{\mathrm{SSDR}}$ & $\widehat{\bar{Y}}_{\mathrm{SSDR}}$ & $\widehat{\bar{Y}}_{\mathrm{SSDR}}$ \\
\hline-0.1 & 100.8797 & 104.1726 & 109.0713 & 118.1939 & 133.1713 & 157.8555 & 191.6527 \\
\hline-0.08 & 100.8806 & 104.1888 & 109.0944 & 118.2593 & 133.3251 & 158.3010 & 192.2725 \\
\hline-0.06 & 100.8808 & 104.2015 & 109.109 & 118.3068 & 133.4430 & 158.6793 & 192.7517 \\
\hline-0.04 & 100.8801 & 104.2107 & 109.1151 & 118.3364 & 133.5250 & 158.9895 & 193.0882 \\
\hline-0.02 & 100.8787 & 104.2164 & 109.1125 & 118.3480 & 133.5709 & 159.2308 & 193.2804 \\
\hline 0 & 100.8764 & 104.2186 & 109.1014 & 118.3416 & 133.5805 & 159.4025 & 193.3276 \\
\hline 0.02 & 100.8733 & 104.2173 & 109.0818 & 118.3172 & 133.5539 & 159.5041 & 193.2295 \\
\hline 0.04 & 100.8694 & 104.2125 & 109.0536 & 118.2749 & 133.4912 & 159.5355 & 192.9865 \\
\hline 0.06 & 100.8646 & 104.2043 & 109.0169 & 118.2147 & 133.3924 & 159.4964 & 192.5998 \\
\hline 0.08 & 100.8591 & 104.1925 & 108.9717 & 118.1366 & 133.2576 & 159.3871 & 192.0710 \\
\hline 0.1 & 100.8527 & 104.1772 & 108.9179 & 118.0408 & 133.0872 & 159.2078 & 191.4025 \\
\hline
\end{tabular}

TABLE 4: Relative efficiencies (in \%) of $\widehat{\bar{Y}}_{\mathrm{LR}}, \widehat{\bar{Y}}_{R}$, and $\widehat{\bar{Y}}_{\mathrm{SSDR}}$ when $n=40$.

\begin{tabular}{|c|c|c|c|c|c|c|c|}
\hline$n=40$ & $\rho=0.1$ & $\rho=0.2$ & $\rho=0.3$ & $\rho=0.4$ & $\rho=0.5$ & $\rho=0.6$ & $\rho=0.7$ \\
\hline$\hat{\bar{Y}}_{\mathrm{LR}}$ & 98.56872 & 101.6871 & 106.8427 & 116.5161 & 131.2267 & 153.2434 & 195.3283 \\
\hline$\overline{\bar{Y}}_{R}$ & 89.4024 & 97.99826 & 107.7805 & 119.1094 & 133.8761 & 150.5256 & 175.4088 \\
\hline$R$ value & $\hat{\bar{Y}}_{\mathrm{SSDR}}$ & $\widehat{\bar{Y}}_{\mathrm{SSDR}}$ & $\widehat{\bar{Y}}_{\mathrm{SSDR}}$ & $\widehat{\bar{Y}}_{\mathrm{SSDR}}$ & $\widehat{\bar{Y}}_{\mathrm{SSDR}}$ & $\widehat{\bar{Y}}_{\mathrm{SSDR}}$ & $\widehat{\bar{Y}}_{\mathrm{SSDR}}$ \\
\hline-0.1 & 100.763 & 104.0057 & 110.1035 & 119.1169 & 133.9862 & 155.4611 & 197.6259 \\
\hline-0.08 & 100.7616 & 104.02 & 110.1465 & 119.1571 & 134.1563 & 155.7931 & 198.4408 \\
\hline-0.06 & 100.7593 & 104.0309 & 110.1807 & 119.2293 & 134.29 & 156.0572 & 199.1088 \\
\hline-0.04 & 100.7563 & 104.0385 & 110.206 & 119.2836 & 134.3872 & 156.2525 & 199.6271 \\
\hline-0.02 & 100.7525 & 104.0426 & 110.2224 & 119.3198 & 134.4476 & 156.3785 & 199.9932 \\
\hline 0 & 100.7479 & 104.0433 & 110.2299 & 119.3379 & 134.4712 & 156.435 & 200.2054 \\
\hline 0.02 & 100.7425 & 104.0406 & 110.2285 & 119.3379 & 134.4579 & 156.4217 & 200.2627 \\
\hline 0.04 & 100.7362 & 104.0345 & 110.2182 & 119.3198 & 134.4078 & 156.3387 & 200.1649 \\
\hline 0.06 & 100.7292 & 104.025 & 110.199 & 119.2837 & 134.3209 & 156.1862 & 199.9125 \\
\hline 0.08 & 100.7214 & 104.0121 & 110.171 & 119.2295 & 134.1974 & 155.9647 & 199.5065 \\
\hline 0.1 & 100.7127 & 103.9959 & 110.134 & 119.1573 & 134.0375 & 155.6746 & 198.9489 \\
\hline
\end{tabular}

TABLE 5: Relative efficiencies (in \%) of $\widehat{\bar{Y}}_{\mathrm{LR}}, \widehat{\bar{Y}}_{R}$, and $\widehat{\bar{Y}}_{\mathrm{SSDR}}$ when $n=60$.

\begin{tabular}{|c|c|c|c|c|c|c|c|}
\hline$n=60$ & $\rho=0.1$ & $\rho=0.2$ & $\rho=0.3$ & $\rho=0.4$ & $\rho=0.5$ & $\rho=0.6$ & $\rho=0.7$ \\
\hline$\hat{\bar{Y}}_{\mathrm{LR}}$ & 99.78738 & 102.7511 & 106.9806 & 115.7236 & 133.4915 & 154.5581 & 191.2787 \\
\hline$\overline{\bar{Y}}_{R}$ & 90.7815 & 98.23825 & 106.5209 & 117.4447 & 135.1337 & 150.7982 & 172.6729 \\
\hline$R$ value & $\widehat{\bar{Y}}_{\mathrm{SSDR}}$ & $\widehat{\bar{Y}}_{\mathrm{SSDR}}$ & $\widehat{\bar{Y}}_{\mathrm{SSDR}}$ & $\widehat{\bar{Y}}_{\mathrm{SSDR}}$ & $\widehat{\bar{Y}}_{\text {SSDR }}$ & $\widehat{\bar{Y}}_{\mathrm{SSDR}}$ & $\widehat{\bar{Y}}_{\mathrm{SSDR}}$ \\
\hline-0.1 & 101.0542 & 104.2512 & 109.2088 & 117.8291 & 135.2587 & 155.6597 & 192.4683 \\
\hline-0.08 & 101.0596 & 104.2689 & 109.2346 & 117.8792 & 135.4549 & 155.9771 & 193.1069 \\
\hline-0.06 & 101.0642 & 104.2832 & 109.2519 & 117.9111 & 135.6138 & 156.2254 & 193.6036 \\
\hline-0.04 & 101.068 & 104.2939 & 109.2606 & 117.925 & 135.7354 & 156.4038 & 193.9563 \\
\hline-0.02 & 101.0711 & 104.3011 & 109.2606 & 117.9206 & 135.8192 & 156.5118 & 194.1633 \\
\hline 0 & 101.0733 & 104.3048 & 109.2521 & 117.8982 & 135.8653 & 156.5493 & 194.2238 \\
\hline 0.02 & 101.0747 & 104.3051 & 109.2349 & 117.8576 & 135.8735 & 156.516 & 194.1373 \\
\hline 0.04 & 101.0754 & 104.3017 & 109.2092 & 117.7989 & 135.8438 & 156.4121 & 193.9044 \\
\hline 0.06 & 101.0753 & 104.2949 & 109.1749 & 117.7222 & 135.7762 & 156.2379 & 193.5261 \\
\hline 0.08 & 101.0743 & 104.2846 & 109.132 & 117.6275 & 135.6709 & 155.9937 & 193.004 \\
\hline 0.1 & 101.0726 & 104.2707 & 109.0806 & 117.515 & 135.5281 & 155.6804 & 192.3406 \\
\hline
\end{tabular}


TABLE 6: Relative efficiencies (in \%) of $\widehat{\bar{Y}}_{\mathrm{LR}}, \widehat{\bar{Y}}_{R}$, and $\widehat{\bar{Y}}_{\mathrm{SSDR}}$ when $n=80$.

\begin{tabular}{|c|c|c|c|c|c|c|c|}
\hline$n=80$ & $\rho=0.1$ & $\rho=0.2$ & $\rho=0.3$ & $\rho=0.4$ & $\rho=0.5$ & $\rho=0.6$ & $\rho=0.7$ \\
\hline$\widehat{\overline{\bar{Y}}}_{\mathrm{LR}}$ & 99.83416 & 102.7266 & 106.6169 & 115.6179 & 131.7743 & 154.1441 & 194.0998 \\
\hline$\overline{\bar{Y}}_{R}$ & 91.45779 & 97.66782 & 105.0443 & 116.7154 & 133.2987 & 150.0163 & 174.2752 \\
\hline$R$ value & $\widehat{\bar{Y}}_{\text {SSDR }}$ & $\widehat{\bar{Y}}_{\text {SSDR }}$ & $\widehat{\bar{Y}}_{\mathrm{SSDR}}$ & $\widehat{\bar{Y}}_{\mathrm{SSDR}}$ & $\widehat{\bar{Y}}_{\mathrm{SSDR}}$ & $\widehat{\bar{Y}}_{\mathrm{SSDR}}$ & $\widehat{\bar{Y}}_{\mathrm{SSDR}}$ \\
\hline-0.1 & 101.3714 & 103.9561 & 108.1498 & 117.1127 & 133.4017 & 154.9814 & 194.8863 \\
\hline-0.08 & 101.3831 & 103.9678 & 108.1557 & 117.1581 & 133.5593 & 155.3240 & 195.5633 \\
\hline-0.06 & 101.3939 & 103.9759 & 108.1534 & 117.186 & 133.6809 & 155.6000 & 196.0938 \\
\hline-0.04 & 101.4039 & 103.9806 & 108.1429 & 117.1966 & 133.7663 & 155.8088 & 196.4753 \\
\hline-0.02 & 101.4131 & 103.9819 & 108.1240 & 117.1897 & 133.8154 & 155.9498 & 196.7060 \\
\hline 0 & 101.4214 & 103.9796 & 108.0970 & 117.1654 & 133.828 & 156.0227 & 196.7849 \\
\hline 0.02 & 101.4289 & 103.974 & 108.0617 & 117.1238 & 133.8042 & 156.0272 & 196.7115 \\
\hline 0.04 & 101.4355 & 103.9648 & 108.0181 & 117.0648 & 133.744 & 155.9634 & 196.4864 \\
\hline 0.06 & 101.4414 & 103.9522 & 107.9664 & 116.9885 & 133.6476 & 155.8314 & 196.1104 \\
\hline 0.08 & 101.4463 & 103.9361 & 107.9065 & 116.8949 & 133.5149 & 155.6315 & 195.5853 \\
\hline 0.1 & 101.4505 & 103.9165 & 107.8385 & 116.7842 & 133.3464 & 155.3643 & 194.9135 \\
\hline
\end{tabular}

TABLE 7: Relative efficiencies (in \%) of $\widehat{\bar{Y}}_{\mathrm{LR}}, \widehat{\bar{Y}}_{R}$, and $\widehat{\bar{Y}}_{\mathrm{SSDR}}$ when $n=100$.

\begin{tabular}{|c|c|c|c|c|c|c|c|}
\hline$n=100$ & $\rho=0.1$ & $\rho=0.2$ & $\rho=0.3$ & $\rho=0.4$ & $\rho=0.5$ & $\rho=0.6$ & $\rho=0.7$ \\
\hline$\overline{\bar{Y}}_{\mathrm{LR}}$ & 100.3458 & 102.4952 & 108.1571 & 117.9797 & 130.9376 & 154.1079 & 194.4502 \\
\hline$\widehat{\bar{Y}}_{R}$ & 90.15273 & 96.99257 & 106.6954 & 119.0344 & 131.7312 & 150.3096 & 174.1364 \\
\hline$R$ value & $\widehat{\bar{Y}}_{\text {SSDR }}$ & $\widehat{\bar{Y}}_{\text {SSDR }}$ & $\widehat{\bar{Y}}_{\mathrm{SSDR}}$ & $\widehat{\bar{Y}}_{\mathrm{SSDR}}$ & $\widehat{\bar{Y}}_{\mathrm{SSDR}}$ & $\widehat{\bar{Y}}_{\mathrm{SSDR}}$ & $\widehat{\bar{Y}}_{\mathrm{SSDR}}$ \\
\hline-0.1 & 101.0155 & 103.6111 & 109.4732 & 119.0467 & 131.8139 & 155.0562 & 195.1446 \\
\hline-0.08 & 101.0193 & 103.6156 & 109.4997 & 119.0954 & 131.9356 & 155.3614 & 195.8685 \\
\hline-0.06 & 101.0224 & 103.6166 & 109.5173 & 119.1661 & 132.0222 & 155.5981 & 196.4477 \\
\hline-0.04 & 101.0246 & 103.6142 & 109.526 & 119.2189 & 132.0735 & 155.7656 & 196.8795 \\
\hline-0.02 & 101.026 & 103.6084 & 109.5259 & 119.2537 & 132.0895 & 155.8634 & 197.1620 \\
\hline 0 & 101.0265 & 103.5992 & 109.5169 & 119.2704 & 132.0701 & 155.8914 & 197.2938 \\
\hline 0.02 & 101.0263 & 103.5865 & 109.499 & 119.2690 & 132.0154 & 155.8494 & 197.2745 \\
\hline 0.04 & 101.0252 & 103.5704 & 109.4722 & 119.2496 & 131.9254 & 155.7375 & 197.104 \\
\hline 0.06 & 101.0233 & 103.5510 & 109.4366 & 119.212 & 131.8303 & 155.5561 & 196.7832 \\
\hline 0.08 & 101.0206 & 103.5281 & 109.3922 & 119.1565 & 131.7603 & 155.3057 & 196.3135 \\
\hline 0.1 & 101.0170 & 103.5018 & 109.3389 & 119.083 & 131.7357 & 154.9868 & 195.6971 \\
\hline
\end{tabular}

TABLE 8: Relative efficiencies (in \%) of $\hat{\bar{Y}}_{\mathrm{LR}}, \hat{\bar{Y}}_{P}$, and $\widehat{\bar{Y}}_{\mathrm{SSDP}}$ when $n=30$.

\begin{tabular}{|c|c|c|c|c|c|c|c|}
\hline$n=30$ & $\rho=-0.1$ & $\rho=-0.2$ & $\rho=-0.3$ & $\rho=-0.4$ & $\rho=-0.5$ & $\rho=-0.6$ & $\rho=-0.7$ \\
\hline$\overline{\bar{Y}}_{\mathrm{LR}}$ & 97.65509 & 100.4233 & 106.4819 & 114.8732 & 127.3161 & 150.3700 & 186.5302 \\
\hline$\overline{\bar{Y}}_{P}$ & 89.13544 & 98.04504 & 108.2301 & 119.1056 & 131.536 & 150.3559 & 172.7989 \\
\hline$R$ value & $\overline{\bar{Y}}_{\text {SSDP }}$ & $\overline{\bar{Y}}_{\text {SSDP }}$ & $\overline{\bar{Y}}_{\text {SSDP }}$ & $\overline{\bar{Y}}_{\text {SSDP }}$ & $\overline{\bar{Y}}_{\text {SSDP }}$ & $\overline{\bar{Y}}_{\text {SSDP }}$ & $\overline{\bar{Y}}_{\text {SSDP }}$ \\
\hline-0.1 & 100.7439 & 104.0964 & 110.2180 & 119.2223 & 131.6342 & 155.0711 & 192.8206 \\
\hline-0.08 & 100.7418 & 104.1117 & 110.2696 & 119.3008 & 131.7849 & 155.371 & 193.4796 \\
\hline-0.06 & 100.7389 & 104.1235 & 110.3126 & 119.3606 & 131.9022 & 155.6019 & 193.9968 \\
\hline-0.04 & 100.7351 & 104.1319 & 110.347 & 119.4016 & 131.9858 & 155.7635 & 194.3700 \\
\hline-0.02 & 100.7305 & 104.1368 & 110.3728 & 119.4239 & 132.0357 & 155.8551 & 194.5975 \\
\hline 0 & 100.7252 & 104.1383 & 110.3899 & 119.4275 & 132.0516 & 155.8766 & 194.6782 \\
\hline 0.02 & 100.719 & 104.1363 & 110.3984 & 119.4122 & 132.0337 & 155.8278 & 194.6118 \\
\hline 0.04 & 100.7119 & 104.1309 & 110.3982 & 119.3781 & 131.9819 & 155.7090 & 194.3986 \\
\hline 0.06 & 100.7041 & 104.122 & 110.3893 & 119.3253 & 131.8963 & 155.5205 & 194.0396 \\
\hline 0.08 & 100.6955 & 104.1096 & 110.3718 & 119.2538 & 131.777 & 155.2627 & 193.5363 \\
\hline 0.1 & 100.6860 & 104.0938 & 110.3456 & 119.1637 & 131.6243 & 154.9363 & 192.8910 \\
\hline
\end{tabular}


TABLE 9: Relative efficiencies (in \%) of $\widehat{\bar{Y}}_{\mathrm{LR}}, \widehat{\bar{Y}}_{P}$, and $\widehat{\bar{Y}}_{\text {SSDP }}$ when $n=40$.

\begin{tabular}{|c|c|c|c|c|c|c|c|}
\hline$n=40$ & $\rho=-0.1$ & $\rho=-0.2$ & $\rho=-0.3$ & $\rho=-0.4$ & $\rho=-0.5$ & $\rho=-0.6$ & $\rho=-0.7$ \\
\hline$\overline{\bar{Y}}_{\mathrm{LR}}$ & 98.81814 & 100.6730 & 107.4055 & 116.5276 & 128.5121 & 148.6026 & 187.6882 \\
\hline$\overline{\bar{Y}}_{P}$ & 91.74324 & 97.31070 & 107.6696 & 119.5246 & 131.7449 & 148.3706 & 171.5955 \\
\hline$R$ value & $\widehat{\bar{Y}}_{\mathrm{SSDP}}$ & $\widehat{\bar{Y}}_{\mathrm{SSDP}}$ & $\widehat{\bar{Y}}_{\mathrm{SSDP}}$ & $\widehat{\bar{Y}}_{\mathrm{SSDP}}$ & $\widehat{\bar{Y}}_{\mathrm{SSDP}}$ & $\widehat{\bar{Y}}_{\mathrm{SSDP}}$ & $\bar{Y}_{\mathrm{SSDP}}$ \\
\hline-0.1 & 101.2921 & 103.7255 & 109.9459 & 119.5251 & 131.8443 & 152.1479 & 190.7650 \\
\hline-0.08 & 101.3028 & 103.733 & 109.9884 & 119.5365 & 131.9973 & 152.3256 & 191.3691 \\
\hline-0.06 & 101.3128 & 103.7371 & 110.0222 & 119.6137 & 132.1165 & 152.4345 & 191.8342 \\
\hline-0.04 & 101.322 & 103.7377 & 110.0472 & 119.6726 & 132.2019 & 152.4742 & 192.1584 \\
\hline-0.02 & 101.3303 & 103.735 & 110.0636 & 119.7132 & 132.2532 & 152.4448 & 192.3400 \\
\hline 0 & 101.3379 & 103.7289 & 110.0711 & 119.7354 & 132.2704 & 152.3461 & 192.3784 \\
\hline 0.02 & 101.3447 & 103.7194 & 110.0700 & 119.7392 & 132.2535 & 152.1787 & 192.2734 \\
\hline 0.04 & 101.3506 & 103.7064 & 110.0601 & 119.7247 & 132.2025 & 151.9428 & 192.0254 \\
\hline 0.06 & 101.3558 & 103.6901 & 110.0415 & 119.6918 & 132.1174 & 151.6391 & 191.6355 \\
\hline 0.08 & 101.3602 & 103.6703 & 110.0141 & 119.6406 & 131.9984 & 151.2684 & 191.1055 \\
\hline 0.1 & 101.3638 & 103.6472 & 109.9781 & 119.5711 & 131.8457 & 150.8318 & 190.4377 \\
\hline
\end{tabular}

TABLE 10: Relative efficiencies (in \%) of $\widehat{\bar{Y}}_{\mathrm{LR}}, \widehat{\bar{Y}}_{P}$, and $\widehat{\bar{Y}}_{\text {SSDP }}$ when $n=60$.

\begin{tabular}{|c|c|c|c|c|c|c|c|}
\hline$n=60$ & $\rho=-0.1$ & $\rho=-0.2$ & $\rho=-0.3$ & $\rho=-0.4$ & $\rho=-0.5$ & $\rho=-0.6$ & $\rho=-0.7$ \\
\hline$\overline{\bar{Y}}_{\mathrm{LR}}$ & 99.45873 & 102.7277 & 107.7386 & 113.9839 & 131.7095 & 153.2816 & 192.1807 \\
\hline$\overline{\bar{Y}}_{P}$ & 90.94703 & 99.18717 & 107.1685 & 115.5287 & 133.5669 & 149.5516 & 173.2163 \\
\hline$R$ value & $\widehat{\bar{Y}}_{\mathrm{SSDP}}$ & $\widehat{\bar{Y}}_{\text {SSDP }}$ & $\widehat{\bar{Y}}_{\mathrm{SSDP}}$ & $\widehat{\bar{Y}}_{\text {SSDP }}$ & $\widehat{\bar{Y}}_{\mathrm{SSDP}}$ & $\widehat{\bar{Y}}_{\mathrm{SSDP}}$ & $\widehat{\bar{Y}}_{\mathrm{SSDP}}$ \\
\hline-0.1 & 101.1575 & 104.661 & 109.4991 & 116.2884 & 133.6678 & 154.36 & 193.0719 \\
\hline-0.08 & 101.1648 & 104.6883 & 109.5359 & 116.3009 & 133.821 & 154.6835 & 193.7006 \\
\hline-0.06 & 101.1714 & 104.7122 & 109.5642 & 116.2955 & 133.9376 & 154.9408 & 194.1851 \\
\hline-0.04 & 101.1771 & 104.7325 & 109.5841 & 116.2724 & 134.0173 & 155.1312 & 194.5234 \\
\hline-0.02 & 101.1821 & 104.7493 & 109.5955 & 116.2316 & 134.0601 & 155.2542 & 194.7139 \\
\hline 0 & 101.1862 & 104.7626 & 109.5984 & 116.173 & 134.0659 & 155.3096 & 194.7557 \\
\hline 0.02 & 101.1895 & 104.7724 & 109.5929 & 116.0968 & 134.0346 & 155.2971 & 194.6486 \\
\hline 0.04 & 101.192 & 104.7786 & 109.5789 & 116.0031 & 133.9663 & 155.2168 & 194.3932 \\
\hline 0.06 & 101.1937 & 104.7813 & 109.5564 & 115.8918 & 133.8612 & 155.0689 & 193.9905 \\
\hline 0.08 & 101.1946 & 104.7805 & 109.5255 & 115.7631 & 133.7193 & 154.8538 & 193.4425 \\
\hline 0.1 & 101.1947 & 104.7761 & 109.4862 & 115.6172 & 133.571 & 154.572 & 192.7515 \\
\hline
\end{tabular}

TABLE 11: Relative efficiencies (in \%) of $\widehat{\bar{Y}}_{\mathrm{LR}}, \widehat{\bar{Y}}_{P}$, and $\widehat{\bar{Y}}_{\text {SSDP }}$ when $n=80$.

\begin{tabular}{|c|c|c|c|c|c|c|c|}
\hline$n=80$ & $\rho=-0.1$ & $\rho=-0.2$ & $\rho=-0.3$ & $\rho=-0.4$ & $\rho=-0.5$ & $\rho=-0.6$ & $\rho=-0.7$ \\
\hline$\widehat{\overline{\bar{Y}}}_{\mathrm{LR}}$ & 100.2239 & 102.4000 & 108.7921 & 116.2027 & 131.1301 & 154.2985 & 190.0055 \\
\hline$\overline{\bar{Y}}_{P}$ & 91.07041 & 96.95651 & 107.8218 & 117.6336 & 132.9936 & 150.1114 & 172.3139 \\
\hline$R$ value & $\widehat{\bar{Y}}_{\text {SSDP }}$ & $\widehat{\bar{Y}}_{\text {SSDP }}$ & $\widehat{\bar{Y}}_{\mathrm{SSDP}}$ & $\widehat{\bar{Y}}_{\mathrm{SSDP}}$ & $\widehat{\bar{Y}}_{\text {SSDP }}$ & $\widehat{\bar{Y}}_{\mathrm{SSDP}}$ & $\widehat{\bar{Y}}_{\text {SSDP }}$ \\
\hline-0.1 & 101.2429 & 103.7526 & 110.1224 & 117.7630 & 133.1038 & 155.1954 & 191.5790 \\
\hline-0.08 & 101.2519 & 103.7577 & 110.1661 & 117.8328 & 133.2751 & 155.5554 & 192.1719 \\
\hline-0.06 & 101.2600 & 103.7593 & 110.2009 & 117.8855 & 133.4115 & 155.8493 & 192.6230 \\
\hline-0.04 & 101.2673 & 103.7574 & 110.2269 & 117.921 & 133.5129 & 156.0764 & 192.9303 \\
\hline-0.02 & 101.2738 & 103.752 & 110.2441 & 117.9394 & 133.579 & 156.236 & 193.0923 \\
\hline 0 & 101.2795 & 103.743 & 110.2523 & 117.9404 & 133.6098 & 156.3278 & 193.1084 \\
\hline 0.02 & 101.2843 & 103.7305 & 110.2516 & 117.9243 & 133.6052 & 156.3516 & 192.9785 \\
\hline 0.04 & 101.2883 & 103.7144 & 110.2421 & 117.891 & 133.5652 & 156.3072 & 192.7031 \\
\hline 0.06 & 101.2915 & 103.6948 & 110.2237 & 117.8404 & 133.4899 & 156.1948 & 192.2835 \\
\hline 0.08 & 101.2939 & 103.6717 & 110.1964 & 117.7727 & 133.3793 & 156.0147 & 191.7216 \\
\hline 0.1 & 101.2954 & 103.6451 & 110.1602 & 117.6880 & 133.2338 & 155.7673 & 191.0199 \\
\hline
\end{tabular}


TABLE 12: Relative efficiencies (in \%) of $\hat{\bar{Y}}_{\mathrm{LR}}, \hat{\bar{Y}}_{P}$, and $\hat{\bar{Y}}_{\text {SSDP }}$ when $n=100$.

\begin{tabular}{lccccccc}
\hline$n=100$ & $\rho=-0.1$ & $\rho=-0.2$ & $\rho=-0.3$ & $\rho=-0.4$ & $\rho=-0.5$ & $\rho=-0.6$ & $\rho=-0.7$ \\
$\widehat{\overline{\bar{Y}}}_{\text {LR }}$ & 100.3811 & 102.6419 & 107.7698 & 118.1677 & 130.9040 & 152.8819 & 196.5500 \\
$\hat{\bar{Y}}_{P}$ & 90.55378 & 97.02887 & 106.4837 & 119.4323 & 131.4591 & 150.0207 & 173.0612 \\
\hline$R$ value & $\hat{\bar{Y}}_{\text {SSDP }}$ & $\hat{\bar{Y}}_{\text {SSDP }}$ & $\widehat{\bar{Y}}_{\text {SSDP }}$ & $\widehat{\bar{Y}}_{\text {SSDP }}$ & $\widehat{\bar{Y}}_{\text {SSDP }}$ & $\widehat{\bar{Y}}_{\text {SSDP }}$ \\
\hline-0.1 & 101.0559 & 103.5352 & 109.2741 & 119.4377 & 131.5400 & 154.2308 & 196.5671 \\
-0.08 & 101.0611 & 103.5394 & 109.2983 & 119.4612 & 131.6587 & 154.4565 & 197.2139 \\
-0.06 & 101.0654 & 103.5402 & 109.3137 & 119.5365 & 131.7426 & 154.6114 & 197.7604 \\
-0.04 & 101.0689 & 103.5377 & 109.3204 & 119.5936 & 131.7916 & 154.6951 & 198.1543 \\
-0.02 & 101.0716 & 103.5318 & 109.3184 & 119.6323 & 131.8055 & 154.7074 & 198.3935 \\
0 & 101.0734 & 103.5226 & 109.3076 & 119.6528 & 131.7843 & 154.6483 & 198.4771 \\
0.02 & 101.0745 & 103.5100 & 109.2881 & 119.6548 & 131.7281 & 154.5179 & 198.4046 \\
0.04 & 101.0748 & 103.4941 & 109.2599 & 119.6385 & 131.6170 & 154.3166 & 198.1764 \\
0.06 & 101.0742 & 103.4748 & 109.2229 & 119.6039 & 131.5610 & 154.0450 & 197.7935 \\
0.08 & 101.0729 & 103.4522 & 109.1772 & 119.5509 & 131.5105 & 153.7037 & 197.2577 \\
0.1 & 101.0707 & 103.4262 & 109.1229 & 119.4797 & 131.4656 & 153.2938 & 196.5715 \\
\hline
\end{tabular}

the numerical simulation comparisons, for example, values of $|\rho|$ : 0.1 (0.1) 0.7. For $\rho$ positive, we compare $\widehat{\bar{Y}}_{R}, \widehat{\bar{Y}}_{\text {SSDR }}$, and $\widehat{\bar{Y}}_{\mathrm{LR}}$, while for $\rho$ negative we compare $\widehat{\bar{Y}}_{P}, \widehat{\bar{Y}}_{\mathrm{SSDP}}$, and $\widehat{\bar{Y}}_{\mathrm{LR}}$.

\section{Results and Discussion}

The results of our simulations are tabulated in the Appendix. For a given value of $n$ the relative efficiencies of $\widehat{\bar{Y}}_{R}, \widehat{\bar{Y}}_{P}$, and $\widehat{\bar{Y}}_{\text {LR }}$ do not depend on $R$; they are, therefore, not included in the main body of the tables but are stated at the top for each value of $\rho$. For $n=30,40,60,80$, and 100 , for each value of $R=0, \pm 0.02, \pm 0.04, \pm 0.06, \pm 0.08$, and \pm 0.10 , we have given the values of $\operatorname{REff}\left(\widehat{\bar{Y}}_{\mathrm{SSDR}}\right)$ for $\rho=0.1,0.2,0.3,0.4,0.5,0.6$, and 0.7 and of $\operatorname{REff}\left(\hat{\bar{Y}}_{\text {SSDP }}\right)$ for $\rho=-0.1,-0.2,-0.3,-0.4,-0.5$, -0.6 , and -0.7 .

As apparent, our proposed estimators $\widehat{\bar{Y}}_{\mathrm{SSDR}}$ and $\widehat{\bar{Y}}_{\mathrm{SSDP}}$ are consistently significantly better than $\widehat{\bar{Y}}_{\mathrm{LR}}$ and $\widehat{\bar{Y}}_{R}$ (or $\widehat{\bar{Y}}_{P}$, as the case may be).

For illustrative purposes, we highlight below the relative efficiency values for the various values of $\rho$, for the cases when $n=30$ and $R=0$. To lessen the obscurity in the results, we have rounded these values to two decimal places. We also include a column for the value of $C=\rho C_{Y} / C_{X}$.

Tables 1 and 2 illustrate very well the relative betterment achieved by our proposed estimators vis-à-vis $\widehat{\bar{Y}}_{\mathrm{LR}}$ and $\widehat{\bar{Y}}_{R}$ (or $\widehat{\bar{Y}}_{P}$, as the case may be). Notably, when $|C|$ is not greater than $1 / 2$, our estimators are more efficient than $\bar{y}$ even though $\hat{\bar{Y}}_{R}$ or $\widehat{\bar{Y}}_{P}$ (as the case may be) is worse than $\bar{y}$ which does not even use auxiliary information. Also when $|C|$ is significantly less than $1 / 2$, our estimators are more efficient than $\bar{y}$ even though
$\widehat{\bar{Y}}_{\text {LR }}$ is worse than $\bar{y}$ (i.e., it fails to use auxiliary information rightly)!

\section{Conclusion}

Our results conform to our motivating aim of achieving more efficient use of auxiliary information. Many other authors, such as Sahai [5] and Chami et al. [6], have suggested efficient variants of ratio and product estimators. In future work we are engaged in comparing these estimators and in trying even better estimators, like the proposed ones, which will be not only more efficient relatively, but also, possibly, more robust against the possible over/underguess of the key-population parameter $C$.

\section{Appendix}

See Tables 3, 4, 5, 6, 7, 8, 9, 10, 11, and 12 .

\section{Conflict of Interests}

The authors declare that there is no conflict of interests regarding the publication of this paper.

\section{References}

[1] P. V. Sukhatme and B. V. Sukhatme, Sampling Theory of Surveys: With Applications, Asia Publishing House, Bombay, India, 2nd edition, 1970.

[2] M. N. Murthy, "Product method of estimation," The Indian Journal of Statistics A, vol. 26, pp. 69-74, 1964.

[3] W. G. Cochran, Sampling Techniques, John Wiley \& Sons, New York, NY, USA, 3rd edition, 1977. 
[4] R Development Core Team, $R$ : A Language and Environment for Statistical Computing, R Foundation for Statistical Computing, Vienna, Austria, 2008.

[5] A. Sahai, "An efficient variant of the product and ratio estimators," Statistica Neerlandica, vol. 33, no. 1, pp. 27-35, 1979.

[6] P. S. Chami, B. Sing, and D. Thomas, "A two-parameter ratioproduct-ratio estimator using auxiliary information," ISRN Probability and Statistics, vol. 2012, Article ID 103860, 15 pages, 2012. 


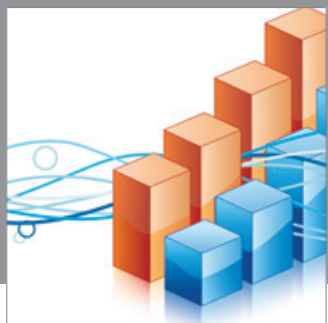

Advances in

Operations Research

mansans

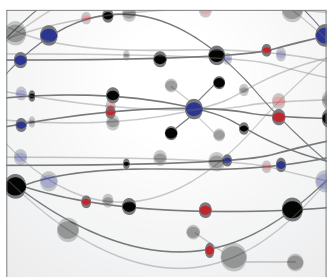

The Scientific World Journal
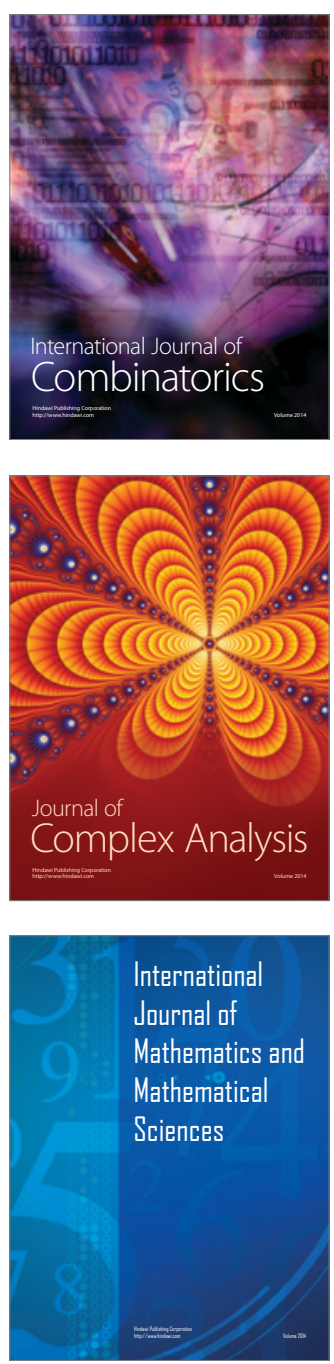
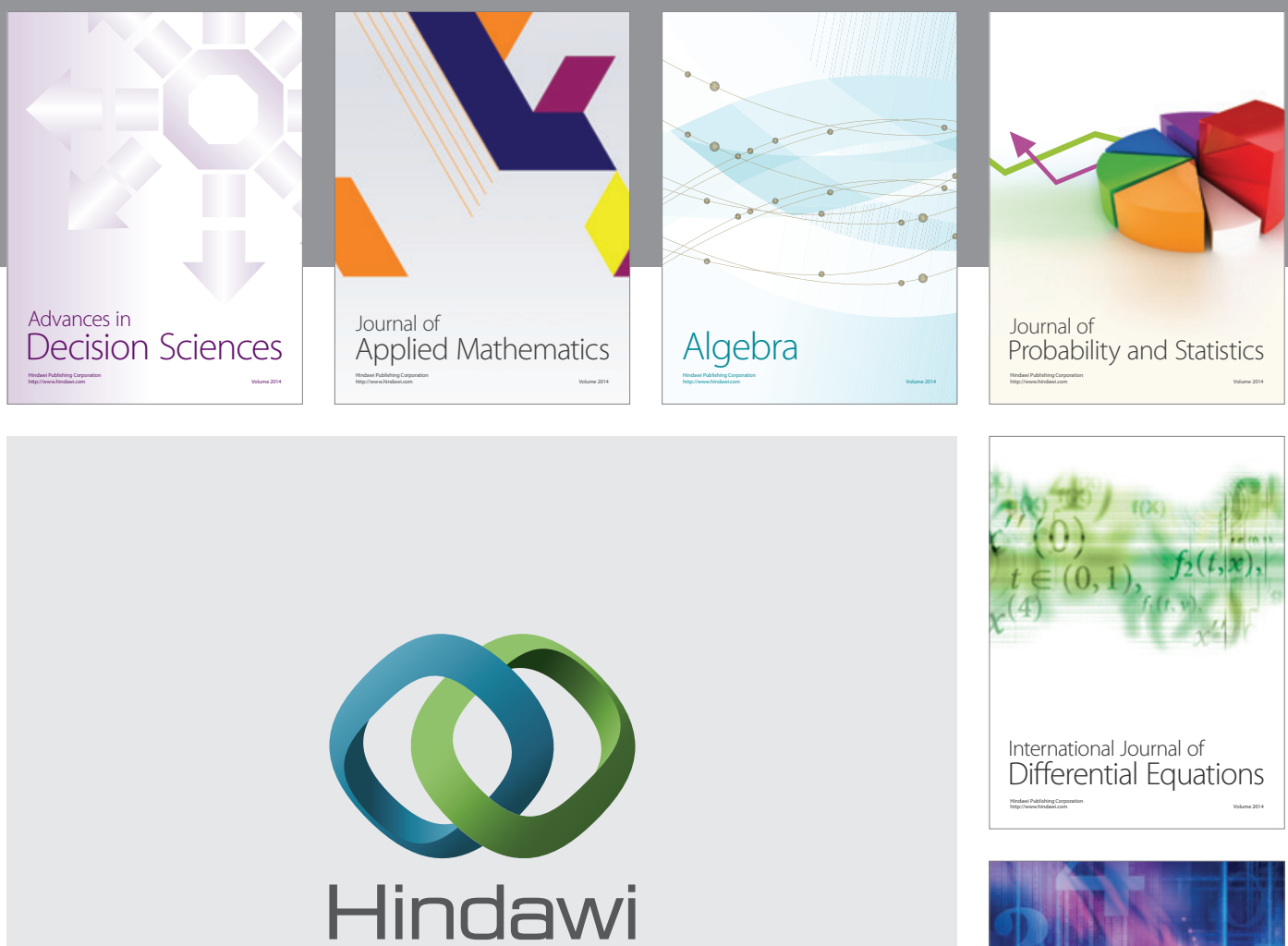

Submit your manuscripts at http://www.hindawi.com
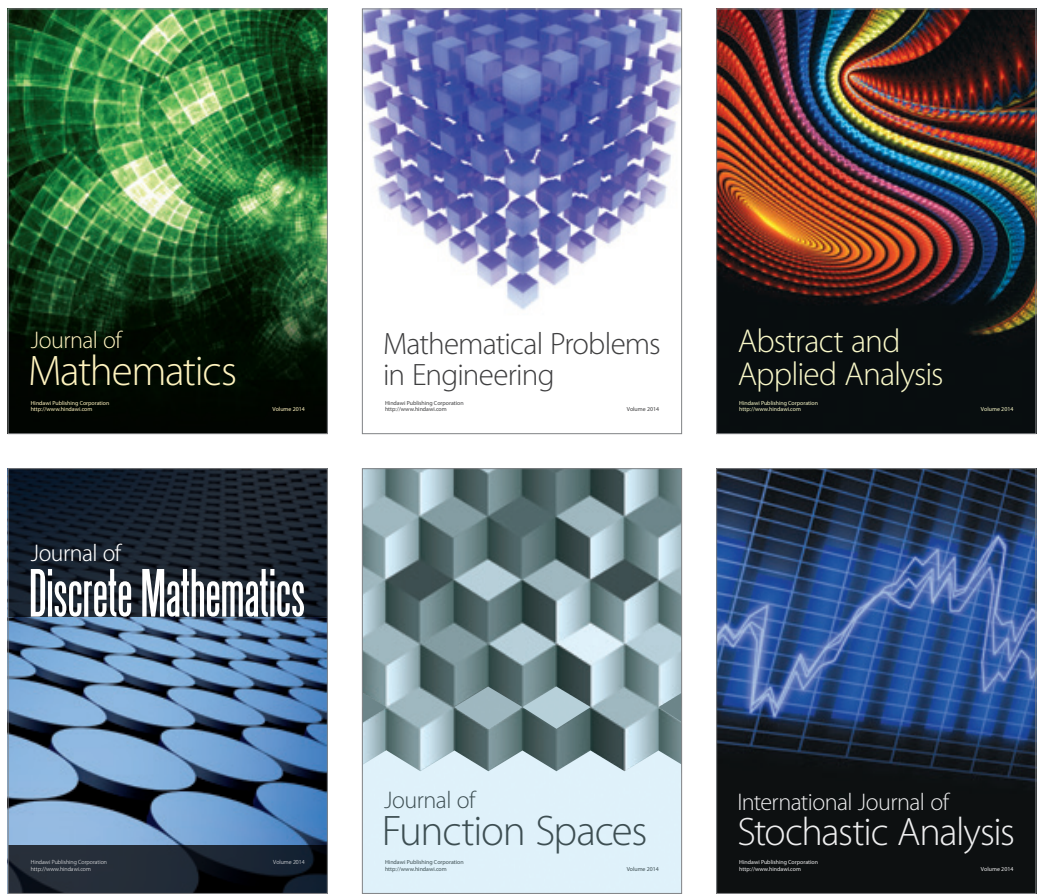

Journal of

Function Spaces

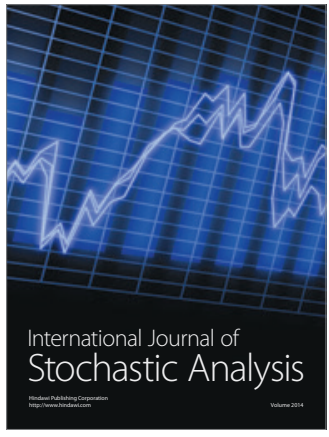

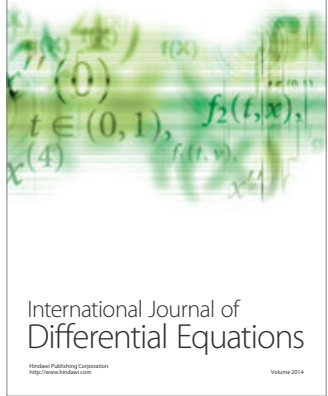
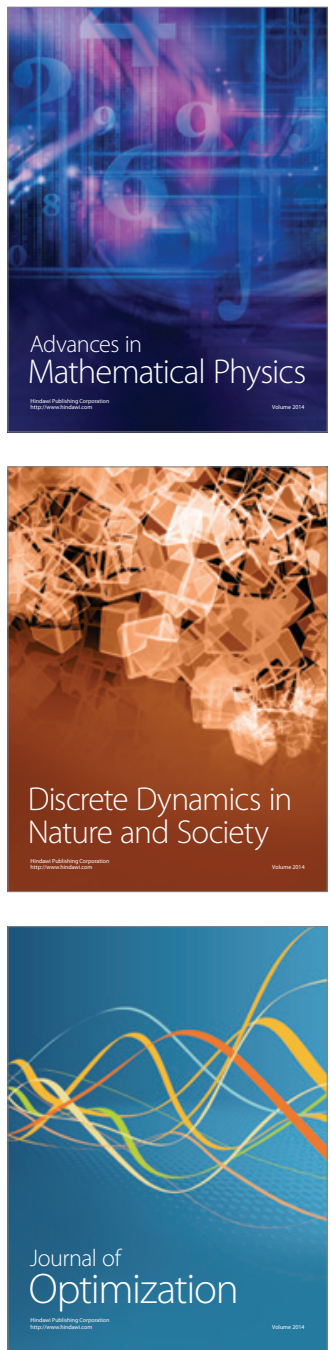